\title{
Photocatalytic Oxidation of Acetic Acid overCdS
}

\author{
Suresh C. Ameta ${ }^{1}$ and Chetna Gomber ${ }^{2}$ \\ ${ }^{I}$ Department of Science, Pacific University, Udaipur-Rajasthan (INDIA) \\ ${ }^{2}$ Department of Chemistry, JIET School of Engineering And Technology For Girls, JIET Universe, N.H-65, Pali \\ Road, Mogra, Jodhpur-Rajasthan(INDIA),
}

\begin{abstract}
The photocatalytic degradation of acetic acid over cadmium sulphidesemiconductor was investigated. The effect of various parameters, such as pH,concentration of acetic acid,amount of catalyst, effect of light intensity were observed. A tentative mechanism has been proposed for the phtocatalyticdegradation of acetic acid using $C d S$ semiconductor as catalyst.
\end{abstract}

Keywords: acetic acid, cadmium sulphide, photocatalytic degradation, semiconductor

\section{Introduction}

Photocatalysis is a research area with a number of fronts. On one hand, it is utilized to synthesize energy rich products, which can serve as synthetic fuels and mineralizing toxic compounds to less toxic or almost harmless species, thus protecting the environment on the other hand. Ordinarily an oxidant is required to oxidize the organic substrates which will leave additional contaminants in the solution after oxidation. In this context, photocatalytic oxidation over semiconducting powders will get an edge over other conventional or classical oxidation reactions as it will not contaminate the solution further.

Wang and Herron [1] studied chemical effects on the optical properties of semiconductor particles. Pichat[2] discussed surface properties, activities and selectivity of bifunctional powder photocatalysts. Ampoet al. [3] observed photocatalytic reactions on native and platinum loaded titanium dioxide. Richard et al.[4] reported that oxidizing species involved in the photocatalytic transformations on zinc oxide is either hole or hydroxyl radicals. de Mayo et al.[5] used CdS supported on a solid surface for catalysis.A kinetic study of photoinduced electron transfer reactions sensitized by colloidal CdS has been reported by Green and Mills [6].

Yoneyamaet al.[7] discussed various factors affecting the product distribution in photocatalytic decomposition of acetic acid on platinized $\mathrm{TiO}_{2}$ in aqueous medium whereas the effects of additives on the reaction was given by Nagashimaet al.[8]Bideauset al.[9] observed the photooxidation of acetic acid by oxygen in presence of $\mathrm{TiO}_{2}$ and dissolved copper ions. Ralmisanoet al.[10] reported the photodecomposition of acetic acid adsorbed over semiconductor and insulator oxide. Kaise et al. [11] reported the photocatalytic reactions of acetic acid on platinum loaded $\mathrm{TiO}_{2}$ and ESR evidence of radical intermediate in the photo-Kolbe reaction.

Awataniet al.[12] studied the in situ infrared spectroscopy of adsorption of lactic acid and related compounds on $\mathrm{TiO}_{2}$ and $\mathrm{CdS}$ semiconductor photocatalyst surfaces from aqueous solutions. A kinetic study with a mechanistically based reaction model for photocatalytic degradation of dichloroacetic acid has been reported by Ballariet al.[13]

Although a lot of work has been carried out in the field of photocatalytic oxidation of various organic compounds, negligible or no information is available on the fate of acetic acid photocatalytic degradation over semiconductor cadmium sulphide powder and therefore, investigations were done to know the details of such degradation.

The effect of various parameters on rate of photocatalytic degradation of acetic acid was observed:

\section{Experimental}

$100.0 \mathrm{~mL}$ of glacial acetic acid was dissolved in $100.0 \mathrm{mLof}$ doubly distilled water to prepare $8.77 \mathrm{M}$ solution, which is then divided into four parts $(50 \mathrm{~mL}$ each) and placed in four beakers.

(i) First beaker containing only acetic acid solution was kept in dark.

(ii)The second beaker containing only acetic acid solution was exposed to light.

(iii) The third beaker having acetic acid solution and $0.12 \mathrm{~g}$ of CdS was kept in dark.

(iv)The fourth beaker, containing acetic acid solution and $0.12 \mathrm{~g}$ of CdS was exposed to light.

After keeping these beakers for three hours, the amount of unreacted acetic acid was measured in each solution. It was observed that there was no or negligible change in the amount of unreacted acetic acid in the first three beakers, whereas in the fourth beaker, the amount of unreacted acetic acid decreases with the increasing period of exposure. This reaction follows a photocatalytic route as it requires light as well as semiconductor CdS, both. 


\section{A Typical Run}

Photocatalytic degradation of acetic acid was observed by taking $8.77 \mathrm{M}$ solution and $0.12 \mathrm{~g}$ of CdS was added to it. This solution was exposed to light intensity $=70 \mathrm{mWcm}^{-2}$ from a 200 Watt tungsten lamp. At regular time interval an aliquot of $2.0 \mathrm{~mL}$ was taken out from the reaction mixture, to which $0.1 \mathrm{~mL}$ ammonium hydroxide and $0.2 \mathrm{~mL}$ of dilute $\mathrm{FeCl}_{3}$ solution was added to develop a red color. The optical density of this red solution was measured with the help of spectrophotometer $\left(\lambda_{\max }=525 \mathrm{~nm}\right)$.

Table .1:A Typical Run. ${ }^{a}$

[Acetic Acid] $=8.77 \mathrm{M}, \mathrm{pH}=3.0$

Light Intensity $=70.0 \mathrm{mWcm}^{-2}, \mathrm{CdS}=0.12 \mathrm{~g}$

Temperature $=308 \mathrm{~K}$

\begin{tabular}{cc}
\hline Time $(\mathbf{m i n})$ & $2+\log$ O.D. \\
\hline 0 & 1.4345 \\
15 & 1.3820 \\
30 & 1.3201 \\
45 & 1.2552 \\
60 & 1.2329 \\
75 & 1.1430 \\
90 & 1.0827 \\
105 & 1.0211 \\
120 & 0.9489 \\
135 & 0.8859 \\
150 & 0.8450 \\
165 & 0.8041 \\
180 & 0.7254 \\
\hline
\end{tabular}

\section{Fig.1: A Typical Run}

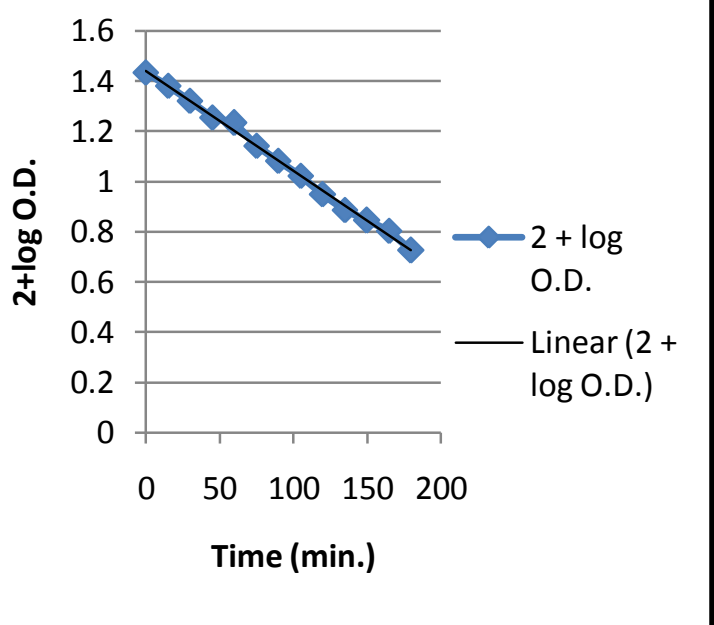

$\mathrm{K}=1.49 \times 10^{-4} \mathrm{sec}^{-1}$

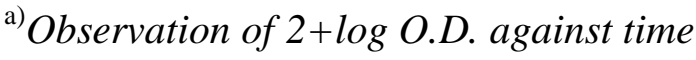

It was observed that the amount of acetic acid decreases with increasing time of exposure. A plot of $2+\log$ O.D. against time was linear and the rate constant was measured by the expression $\mathrm{k}=2.303 \mathrm{x}$ slope. The results are reported in the Table.1. and represented by graph in Fig .1.

\section{Mechanism}

On the basis of the observations, a tentative mechanism has been proposed for the photocatalytic degradation of acetic acid in the presence of semiconducting $\mathrm{CdS}$ powder.

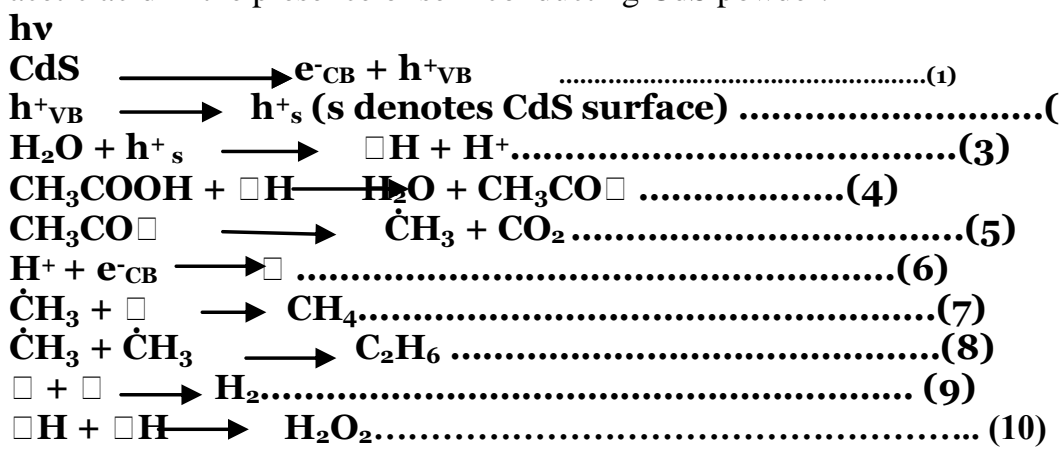

On exposure of $\mathrm{CdS}$ particles, an electron from the valence band is excited to conduction band $\left(\mathrm{e}_{\mathrm{CB}}^{-}\right)$leaving behind a hole in the valence band $\left(\mathrm{h}^{+}{ }_{\mathrm{VB}}\right)$. This hole creates electron deficiency $\left(\mathrm{h}_{\mathrm{S}}^{+}\right)$at semiconductor surface [14], which can dissociate water into hydroxyl radical and a proton [15]. This hydroxyl radical abstracts a hydrogen from acetic acid forming acetate radical which may further decarboxylate to generate methyl radical. There is a possibility of hydrogen radical generation by combination of a proton with an electron in the conduction band and this hydrogen radical can combine with methyl radical to form methane gas; however, the possibility of dimerization of methyl, hydrogen and hydroxyl radicals to give ethane, hydrogen and hydrogen peroxide, respectively, cannot be ruled out. 
The formation of carbon dioxide was detected by its usual test with lime water. A new Kolbe reaction pathway for heterogeneous photocatalytic synthesis of methane from $\mathrm{CH}_{3} \mathrm{COOH}$ has been reported by Kraeutler and Bard [16, 17].

The reaction was also carried out in the presence of hydroxyl radical scavenger like 2-propanol and it was observed that the rate of photocatalytic degradation of acetic acid was drastically reduced: hence, confirming the participation of hydroxyl radical as an active oxidizing species.

\section{References}

[1] Y. Wang and Herron, J. Phys. Chem., 91, 1987, 5005

[2] P. Pichat,,New J. Chem., 11, 1987, 135

[3] M. Anpo, T. Shima, S. Kodama, A.J. Amass and M. Kulokawa, J. Phys. Chem., 91, 1987, 4305

[4] C. Richard, P. Boule and J. M. Aubry, J. Photochem. Photobiol., 60A, 1991, 235

[5] P. de Mayo, K. Muthurama and K. W. Wun, Catal. Lett., 10, 1991, 71

[6] A. Green and A. Mills, J. Photochem. Photobiol., 64A, 1992, 211

[7] H. Yoneyama, Y. Takao, H. Tamura and A.J. Bard, J. Phys Chem., 87,1983, 1417

[8] T. Nagashima, K. Hirano, Y. Asami and R. Takagi, Nippon Kagaku Kaishi, 1988, 1232

[9] M. Bideaus, B. Claudel, L. Faure and H. Kazouan, J. Photochem. Photobiol., 61A, 1991, 269

[10] L. Ralmisano, M. Schiovello, A. Sclafani S. Coluccia and P.L. Marcheel, New J. Chem., 12, 1980, 847

[11] M. Kaise, H. Kondoh, C. Nishihara, H. Nozoy, H. Shindo, S. Nimura and O. Kibuchi, J. Chem. Soc. Chem. Comm., 1993, 395

[12] T. Awatani, K. D. Dolison, A.J. McQuillan, B. Ohtani and K. Osaki, Chem. Lett., 8, 1992, 849

[13] Maria de los Milagros Ballari, Orlando O. Alfano and Alberto E. Cassano, Ind. Eng. Chem. Res., 48(4), 2009, 1847-1858

[14] Y.Wu, Y. Li and Q. Zhuang, J. Photochem. Photobiol., 62 A, 1991, 261

[15] T. Sakata, T. Kawai and K. Hashimota, J. Phys. Chem., 88, 1984, 2344

[16] B. Kraeutler and A.J. Bard, J. Amer. Chem. Soc., 100, 1978, 2239

[17] B. Kraeutler and A.J. Bard, J. Amer. Chem. Soc., 100, 1978, 5985 